CASE REPORTS

\title{
Treatment of permanent pacemaker electrode-induced cardiac perforation
}

\author{
Yongdong Li,; Zhiping Ge, Lina Wei, Songkui Dai \\ The Third Affiliated Hospital of Inner Mongolia Medical University, Baotou, China
}

Received: May 20, 2015

DOI: $10.14725 /$ dcc.v2n3p34
Accepted: July 28, $2015 \quad$ Online Published: September 10, 2015

URL: http://dx.doi.org/10.14725/dcc.v2n3p34

\begin{abstract}
A case of permanent pacemaker electrode-induced perforation in the Third Affiliated Hospital of Inner Mongolia Medical University was collected and analyzed on the basis of diagnosis, physical examination and treatment. Cardiac perforation is one of the most serious complications of pacemaker implantation. As this disease is rare, it is easy to be misdiagnosed or missed diagnosis. We hope to improve the clinician's awareness and vigilance of permanent pacemaker electrode perforation by analyzing the data of this case.
\end{abstract}

Key Words: Pacemaker, Electrode perforation

\section{Medical record}

\subsection{General information}

A 79-year-old male was admitted to our department on May 20, 2010 due to repeated dizziness, fatigue for five years' duration, aggravating with syncope 4 times in one year. The patient presented with dizziness, fatigue five years ago, without chest tightness, chest pain, or paroxysmal nocturnal dyspnea. The symptoms have not been taken seriously and the patient was not given any treatment. In the past year, the symptoms increased and syncope occurred 4 times. There were no obvious causes, and each attack lasted 1-2 min, the patient turned to be conscious of himself afterwards. Since the onset, the spirit and diet are normal with no change in weight. History of hypertension or diabetes was denied.

\subsection{Physical examination}

$\mathrm{T} 36.5^{\circ} \mathrm{C}, \mathrm{P} 48$ beats $/ \mathrm{min}, \mathrm{R} 16 / \mathrm{min}$, blood pressure $110 / 80 \mathrm{mmHg}$. The patient was conscious with an active position. The breathing was smooth and the chest was sym- metric. Double lung breath sounded resonance, and no dry or wet rales were heard. The heart rate was 48 beats/min, showing regularity in the force and rhythm of the heartbeat. No noises were heard in each valve during auscultation. Abdomen soft, mild epigastric tenderness, without rebound tenderness and muscle tension. No enlargement of liver, spleen and kidneys beneath the rib was found, nor edema of lower limbs.

\subsection{Auxiliary examination}

Routine blood test, WBC: $7.4 \times 10^{9} / \mathrm{L}$, Pt: $346 \times 10^{9} / \mathrm{L}$, $\mathrm{Hb}: 12.84 \mathrm{~g} / \mathrm{L}$. Biochemistry, $\mathrm{K}^{+}: 4.12 \mathrm{mmol} / \mathrm{L}, \mathrm{Na}^{+}$: $138.5 \mathrm{mmol} / \mathrm{L}, \mathrm{Cl}^{-}: 100 \mathrm{mmol} / \mathrm{L}, \mathrm{GLU}: 4.80 \mathrm{mmol} / \mathrm{L}, \mathrm{T} 3$, T4, TSH were normal. ECG after admission showed: sinus rhythm, sinus arrest, $4.0 \mathrm{~s}$. Holter showed: sinus rhythm, sinus arrest $5.44 \mathrm{~s}$, the average heart rate of 42 beats $/ \mathrm{min}$, paroxysmal atrial tachycardia. Echocardiography showed: left atrium $39 \mathrm{~mm}$, left ventricular $48 \mathrm{~mm}$, interventricular septal $8 \mathrm{~mm}$, left posterior wall $8 \mathrm{~mm}$, EF $58 \%$, right atrium $38 \mathrm{~mm}$, right ventricle $23 \mathrm{~mm}$. No abnormalities were found

\footnotetext{
*Correspondence: Yongdong Li; E-mail: doctoryongdong@sina.com; Address: The Third Affiliated Hospital of Inner Mongolia Medical University, Baotou, China.
} 
in cranial CT.

\subsection{Primary Diagnosis}

Sick sinus syndrome (slow-fast type).

\subsection{Diagnosis and treatment}

The patient had a permanent pacemaker implanted in class I indications. For economic reasons, the patient was given implantation of ventricular single chamber pacemakers (VVI). Permanent pacemaker implantation was performed on the third day after admission. The operation was successful. The pacing threshold was $0.3 \mathrm{~V}$, the impedance was $536 \Omega$, the $\mathrm{R}$ wave height was $9.8 \mathrm{mv}$, and the pacing was normal. The pacing ECG was shown in Figure 1, and the intraoperative image was shown in Figure 2. The patient took out-of-bed activity at $24 \mathrm{~h}$ after operation, and the ECG monitoring showed that the pacing perception function was normal. On the third day of operation, the pacemaker performed pacing disturbance. The pacing signal showed no QRS wave group and was normal (see Figure 3). The patient had no chest pain, abdominal pain, palpitation nor shortness of breath. $X$-ray examination showed that the electrode was located at the apex of the right ventricle (see Figure 4). No pericardial effusion was found over echocardiography. Above mentioned conditions concluded pacing, micro dislocation of electrode or perforation of my- ocardium. The pacemaker test failed to pace at the voltage of $6.9 \mathrm{~V}$. However, when the polarity of pacing electrode was changed from unipolar to bipolar, pacemaker function was normal, pacing threshold was $3.0 \mathrm{~V}$, pacing mode was VVI. It was mainly because of pacing electrode perforation induced-circumpolar pacing. The pacing voltage was adjusted to $4.2 \mathrm{~V}$, and the ECG monitoring showed that the pacing function was normal and the pacing mode was VVI, as shown in Figure 5. On the third day after adjustment of the parameters, the ECG of the patient monitored at the bedside showed pacing disturbance, pacing signals were found with no QRS wave group, as shown in Figure 6. The patient did not have any self-conscious symptoms. $X$-ray examination: electrode location in the heart margin of the apex (see Figure 7). Re-implantation of pacing electrode was operated. Operation procedure: a new active spiral electrode was implanted into the right ventricular septum, the pacing threshold was $0.4 \mathrm{~V}$, the impedance was $506 \Omega$, the $\mathrm{R}$ wave height was $19.8 \mathrm{mv}$, and the pacing was normal, as shown in Figure 8. In the fluoroscopy, the original electrode was pulled out, and the changes of heart shadow and heart beat were carefully observed during the operation so as to prevent the occurrence of pericardial effusion. And early warning of cardiac tamponade after opening chest preparation was taken, under heart surgery coordination. $20 \mathrm{~min}$ after operation, heart beat and heart shadow were normal seen in Figures 9 and 10. Ultrasound showed no effusion during bedside examination. The operation went smoothly.

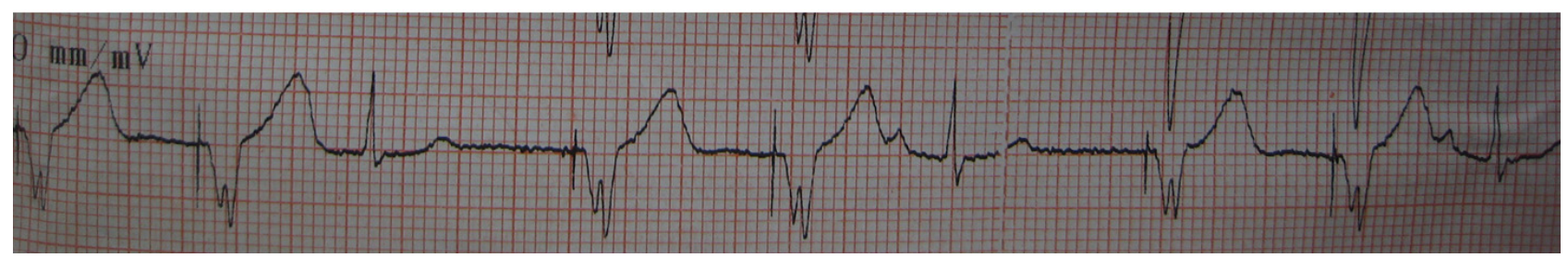

Figure 1: Pacing ECG

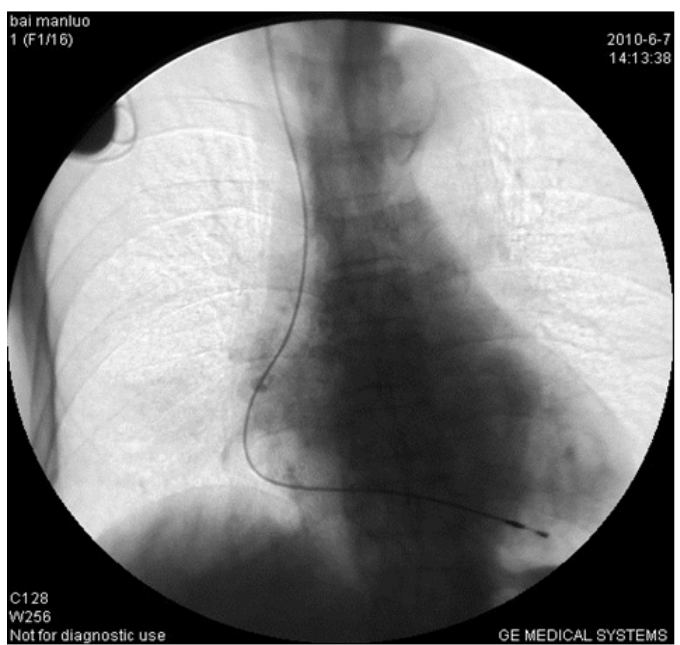

Figure 2: Posteroanterior radiograph

\section{Discussion}

\subsection{Dr. Lina Wei}

Lina Wei, a chief physician of Department of Cardiology at the Third Affiliated Hospital of Inner Mongolia Medical University, specializing in cardiac pacing and electrophysiology.

Cardiac perforation is one of the severe complications caused by pacemaker electrode, occurring during pacemaker implantation even several years after the surgery. The clinical manifestation is diversified according to the perforation position as well as the position of the end of the electrode wire. Generally, most of the cardiac perforation can be asymptomatic or showing the pacing and/or perceptual dysfunction only, which is difficult to be found clinically. The patient in our case had no obvious symptoms. The cardinal 
symptom of typical cardiac perforation includes chest pain or local muscle beating, such as intercostal muscles, mediastinum and diaphragm due to electrode sting. Clinically, cardiac tamponade indicates the appearance of palpitation, shortness of breath, cyanosis, dysphoria and other clinical manifestations. Specific performance is as follows:

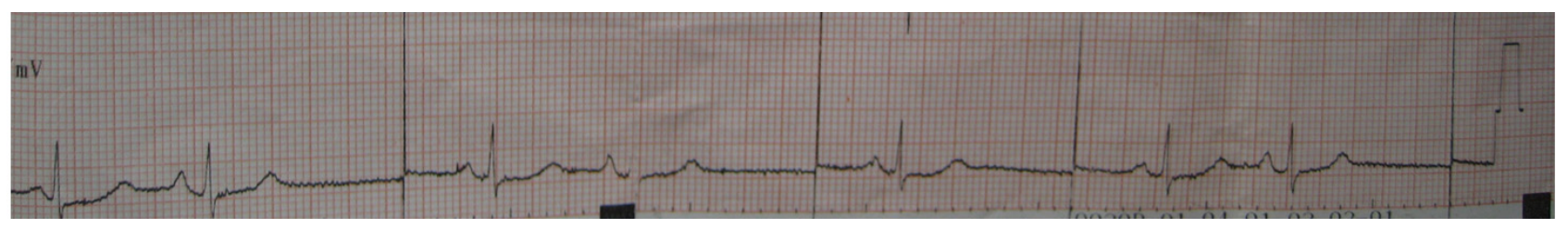

Figure 3: ECG monitoring diagram on the third postoperative day

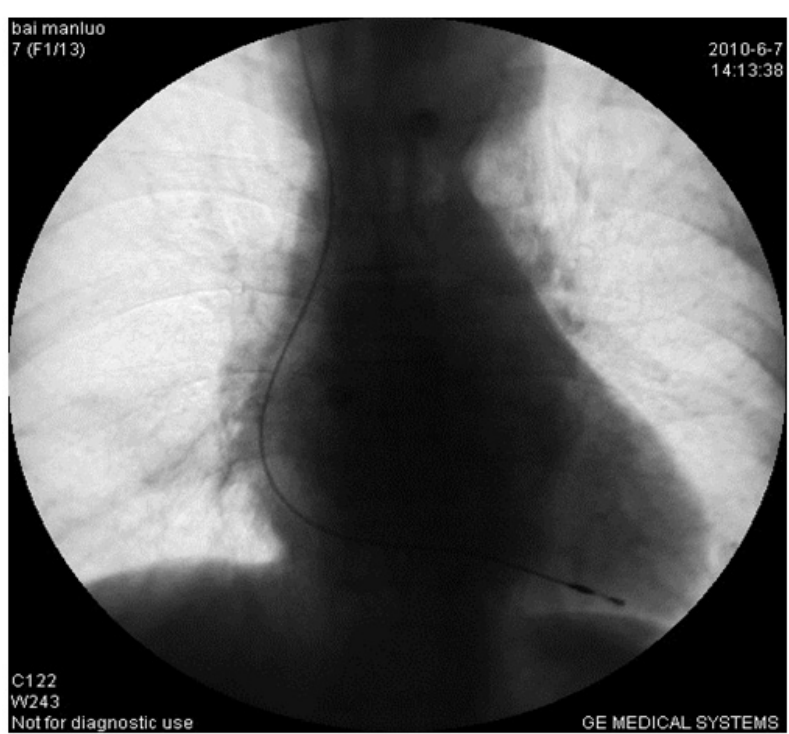

Figure 4: Posteroanterior radiograph

(1) Pacing and/or perceptual dysfunction. Electrode perforation is associated with the dysfunction even the loss of pacing and/or percept, in which pacing function is firstly involved. The ECG of the patient with myocardial perforation showed poor pacing function with no obvious symptoms. The sudden loss of pacing function due to electrode perforation would lead to death of a complete pacemaker dependent patients (such as three degree atrioventricular block and a low ventricular rate). There is a risk of triggering a malignant arrhythmia among some patients with underlying heart disease because of perceptual dysfunction. Of the 5 cases of chronic heart perforation reported by Polin GM et al., ${ }^{[1]}$ two of them had chronic perforation and were first diagnosed by abnormal pacing and sensory testing during long-term follow-up. Other reports have found that most electrode wires led to pacing, especially in patients with no pericardial effusion, despite the increased pacing threshold.

(2) Chest pain, abdominal pain, diaphragm stimulation. Some patients with heart perforation had chest pain, abdominal pain, or diaphragm stimulation. Generally the sharp pain is occured and is associated with or- ganic heart disease. The electrode perforation or pericardial effusion may not be discovered in chest $X$-ray when the symptoms began to appear.

(3) Pneumothorax, hemothorax, hemopneumothorax and pneumomediastinum. It is likely to cause pneumothorax, hemothorax, hemopneumothorax and pneumomediastinum if electrode wire penetrates into the atrial wall to lung or pleural cavity.

(4) Pericardial effusion. Some patients with electrode perforation would suffer from pericardial effusion. However, in the early stage of chest pain, the volume of fluid is not large, and with the increase in the amount of fluid in the later period, there will be abnormal hemodynamics, such as cardiac tamponade and cardiogenic shock. In this case, pericardial ultrasound was performed without any detection of pericardial effusion, which was associated with the involvement of early intervention of myocardial perforation.

(5) Cardiac tamponade. There were cardiac tamponade and cardiogenic shock in patients who were not treated in time. Comparing with general cardiac tamponade, pacing lead related cardiac tamponade carries a higher mortality rate. It occurs frequently during or after surgery. If the pressure of atrial electrode perforation is not large, the blood will slowly seep into the pericardial cavity, which can cause late cardiac tamponade. One patient was reported to have chest pain after pacemaker surgery for 30 days, and a small pericardial effusion was detected by ultrasound. After two weeks, the patient developed symptom of shortness of breath. Echocardiography examination prompted the massive pericardial effusion. Hence, we had a close observation of the implantation and postoperative follow-up of the pacemaker, with the possibility of an inadvertent accumulation of fluid.

\subsection{Dr. Songkui Dai}

Songkui Dai, the head of Department of Cardiology at the Third Affiliated Hospital of Inner Mongolia Medical University, specializing in clinical diagnosis and treatment of coronary heart disease. 
The definition and classification of myocardium perforation caused by pacemaker implantation: early and late cardiac perforation are differentiated by the length of pacemaker lead perforation and pacemaker implantation time. It is called early perforation when it occurs within first months after the operation. The perforation, which is more than one month after the surgery, is called advanced perforation. Cardiac perforation after pacing is a cardiac perforation associated with cardiac pacing lead, which is mainly involved in perforation of atrial and ventricular muscles during or after pacemaker surgery. Cardiac perforation in the broad sense also includes three pacemaker related tricuspid, mitral valve perforation and automatic implantable cardiac defibrillators (ICD), myocardial wall or coronary sinus perforation related to cardiac resynchronization pacing (CRT), and cardiac perforation of the pacing lead of right ventricular to the left, by way of atrial septal and mitral valve. Most heart perforation cases are due to the operation of cardiac electrode leads, so cardiac perforation occurs more frequently during or after surgery. Some patients had no obvious signs of electrode lead perforation during the procedure. For example, the patient's intraoperative perception of pacemaker and pacing function were normal, $X$-ray images were not specific, but myocardial perforation occurred third days after surgery.

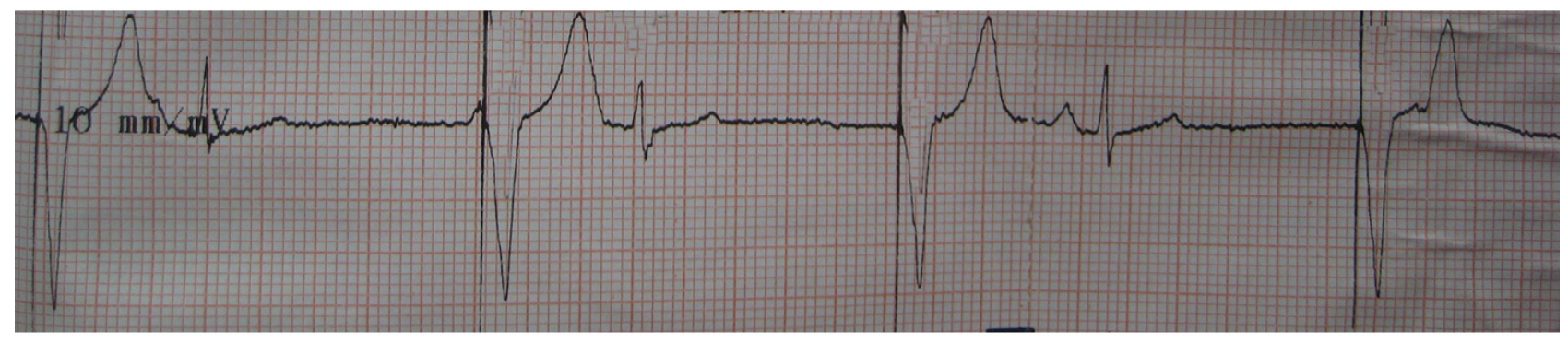

Figure 5: ECG monitoring diagram with pacing electrode

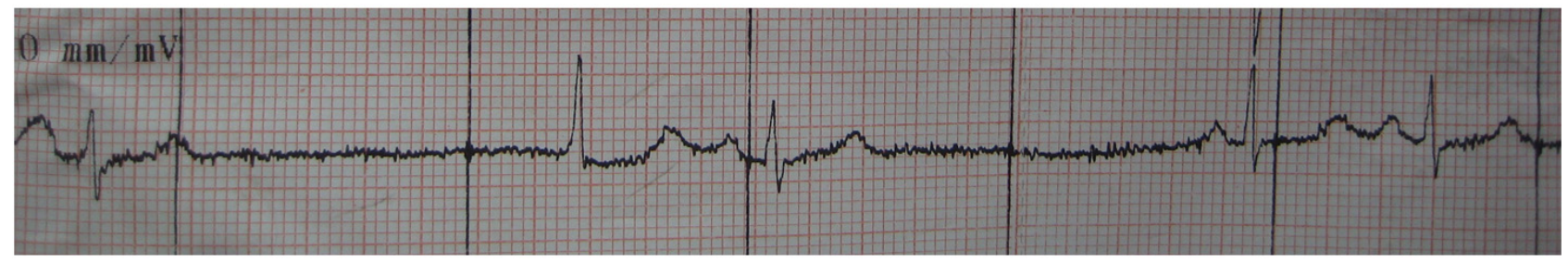

Figure 6: ECG monitoring diagram at the third day after adjustment of the parameters

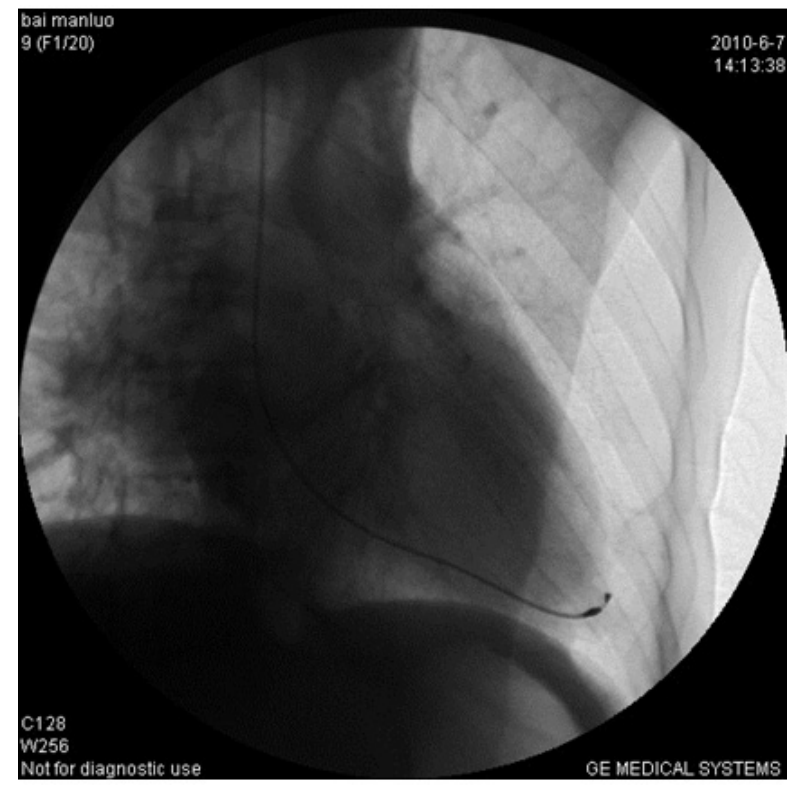

Figure 7: Posteroanterior radiograph
The incidence of cardiac perforation after pacemaker implantation: the incidence of heart perforation at early stage was $5 \%-7 \%$, which was related to the hardness of electrode wire and the lack of experience. With the application of modern pacing lead and the popularization of implantation technology, the complications of cardiac perforation have decreased obviously. According to a retrospective analysis of the Mayo heart center in 2006, the total incidence of cardiac perforation was $1.2 \%$ in 4,280 patients. Early heart perforation was mostly sporadic cases. Polin GM et al. ${ }^{[1]}$ summarized the chronic cardiac perforation for more than 30 days. 2,535 consecutive patients were followed up for 3 years. 5 patients with perforation of heart greater than 30 days were found, with a rate of $0.17 \%$. For the difference in the incidence of heart perforation between passive and active fixation electrodes, most authors believed that the use of ventricular active electrodes could reduce the occurrence of myocardial perforation. The incidence of complications could be as high as $9 \%$, especially when the electrode wires were placed on the right atrial appendage or anterior wall of 
the atrium.

Pacemaker implantation induced cardiac perforation: it occurs during surgery as a result of a rough operation, especially when inserting a hard lead wire or hard temporary pacing electrode. It is related to the insufficient understanding of $X$-ray projection, as well as the age and the preoperative application of hormones. Middle and late perforation are often caused by too large tension of electrode wire and too tight myocardium. Perforation usually occurs on the right ventricular surface when the electrodes enter the pericardial cavity or reach the diaphragm, or there is perforation of the interventricular septum or coronary sinus. The myocardial perforation in this patient was related to the high tension of the right ventricular electrode. In general, permanent pacemaker and ICD atrial and ventricular lead wires are placed in the right atrium or right ventricle respectively, and the left ventricular pacing electrode of CRT is often placed in the coronary sinus. Under normal circumstances, the left and left ventricular wall thicknesses are $3 \mathrm{~mm}$ and 12-15 mm, respectively. Compared with the left ventricular system, the right and right walls are thinner, $2 \mathrm{~mm}$ and 3-4 mm, respectively. The thickness of the right walls is less than $1 / 3$ of the left, and the wall of the right ventricu- lar apex is thinnest and the most vulnerable part of cardiac perforation. $^{[2]}$ In addition, the thin coronary sinus is also a predilection site for perforation. Common causes of cardiac perforation after pacemaker implantation include: the use of hormones once or now, active fixation electrode or temporary pacing electrode, the electrode wire for adults used by children, tighter local tension caused by long reserved lead, bipolar electrodes lead implanted in patients with body mass index $<20$, and so on. These reasons may increase the risk of heart perforation. ${ }^{[3]}$ Our patient had a body mass index of 18 in short stature and slender, and carried a high risk of perforation, suggesting that the right ventricular electrodes should be maintained with proper tension for this type of patient. In addition, compared with unipolar wires, bipolar wires are more prone to heart perforation due to their thicker diameter and stiffer texture. The electrode tip surface area of small electrode wires leads to cardiac perforation due to local pressure; Too long implantation time caused by lack of experience, deformity of the chest and vessel during operation may also increase the possibility of perforation of the lead through the ventricular electrode. Studies have shown that up to $16.9 \%$ of heart perforations can be caused by improper medical operation. ${ }^{[4]}$

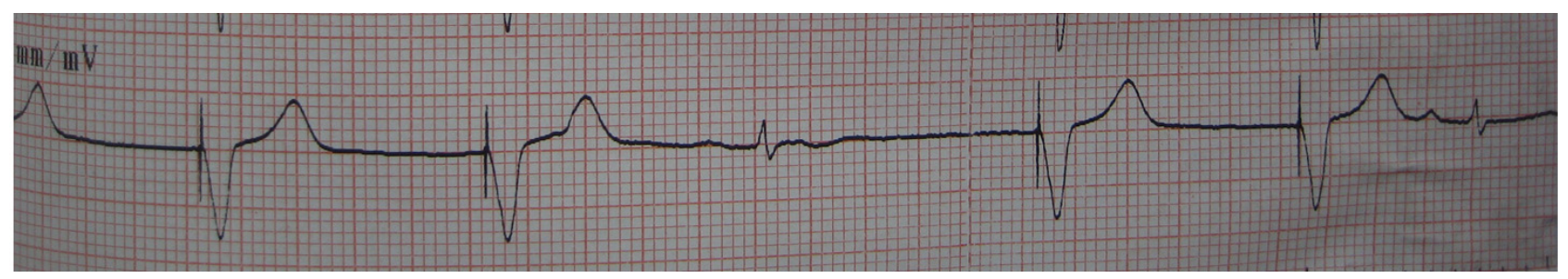

Figure 8: ECG monitoring diagram after re-implantation surgery of pacing electrode

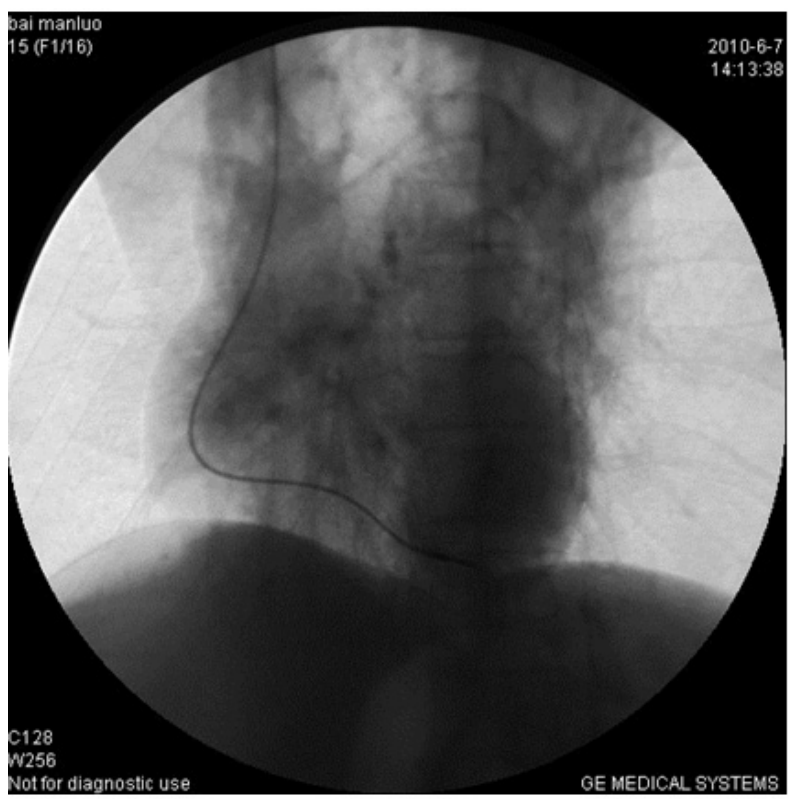

Figure 9: Left anterior oblique

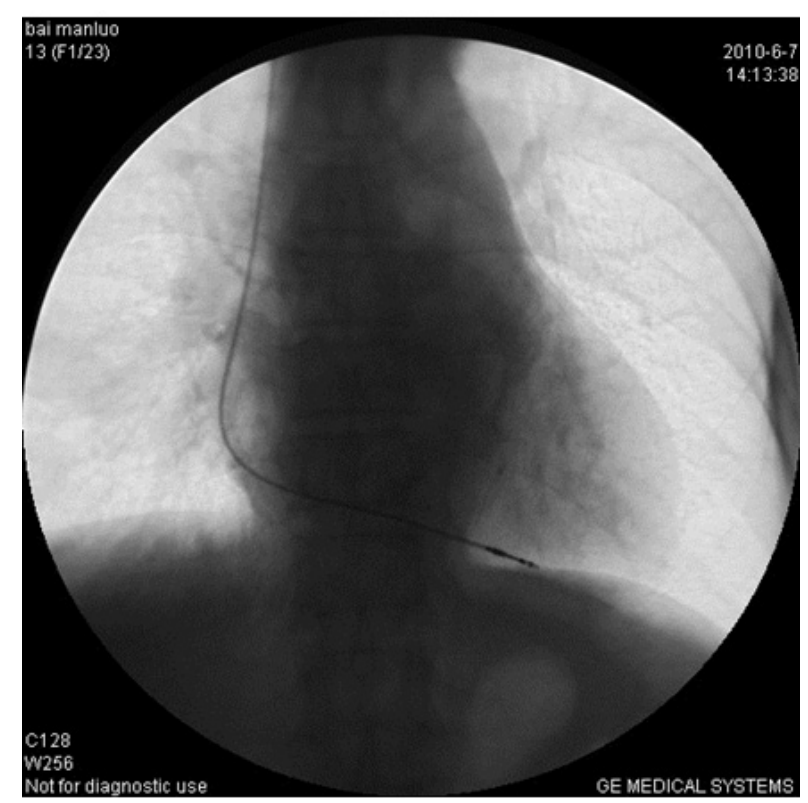

Figure 10: posteroanterior radiograph 


\subsection{Dr. Zhiping Ge}

Zhiping Ge, a professor at the Third Affiliated Hospital of Inner Mongolia Medical College, specializing in coronary heart disease, interventional therapy and cardiac pacing and electrophysiology.

The premise of correct diagnosis is the recognition and attention of this complication. Diagnosis can be made based on symptoms, signs, chest $X$-ray, echocardiography, multi-slice helical CT, and pacemaker tests. When the pacemaker is implanted for one week or several months, the disease could be suspected if the symptoms of chest pain occur. The examination of the electrocardiogram, chest $X$-ray or pacemaker function is available for correct diagnosis. Most patients with cardiac perforation are asymptomatic, and some may present with chest pain, abdominal pain, and diaphragm irritation. There are several signs: cardiac perforation features suggestive of electrode wire, precordial pain, pericardial friction sound. Auxiliary examination: ECG: the left bundle branch block pacing pattern changes into right pattern. It is a typical ECG change from right to left ventricle. Atrial electrode perforation leads to pacing extreme changes. The most evident ECG performance is the atrial channel pacing and perception dysfunction or loss as the atrial depolarization wave is smaller. In addition, atrial conduction delay can also be demonstrated. Echocardiography: echocardiography is not only helpful to identify whether the heart electrode leads to the heart, but also to find the amount of pericardial effusion and the amount of fluid accumulated. $X$-ray examination: it is the most simple and significant means to identify cardiac perforation. Perforation of different degrees presents different features. After the perforation of the right ventricular apex, some patients can not be diagnosed only in the posterior position, but the diagnosis is made when the left and right anterior oblique angles are used. The electrodes on the right outer margin of the heart could be seen in the patient under frontal $X$-ray examination. CT examination: it is suitable for the cases that routine ECG, $X$-tay and cardiac ultrasound failed to diagnose, and ultimately relied on 64-slice spiral CT. ${ }^{[5]}$ Three dimensional color ultrasound can be used in patients who have not been diagnosed with CT. In addition, right ventricular angiography and single level electrogram examination are helpful to detect cardiac perforation. ${ }^{[6]}$

\subsection{Dr. Yongdong Li}

Yongdong Li, a chief physician at the Third Affiliated Hospital of Inner Mongolia Medical College, specializing in implantation of permanent pacemaker and interventional therapy of coronary heart disease.

The core issue in the treatment of cardiac perforation associated with pacing electrode leads is the management of the electrode wires. How to deal with it? How to treat with pericardial effusion? The general principles of treatment include the following factors:

(1) The management of electrode lead. Pacing electrode wires in patients with early cardiac perforation need to be removed and replaced, followed by regular cardiac ultrasound follow-up. Most cardiac perforation cases carry good prognosis so that surgical thoracotomy is not required. The general conservative treatment is to remove electrode lead. Supportted by ultrasound and cardiac surgery, place another ventricular pacing lead, then remove the perforation electrode wire under ultrasound examination and cardiac surgery, in order to avoid the occurrence that the new implanting electrodes may pierce the heart through the original breach. ${ }^{[7]}$ While, transvenous lead extraction imposes a hazard to patients with chronic perforation since electrode wire are bound solidly to myocardial tissues. ${ }^{[8]}$

(2) The management of pericardial effusion. Pericardiocentesis should be performed immediately when a large amount of pericardial effusion appears, especially in those with cardiac tamponade. Some patients treated with pericardiocentesis are found to recover well without any abnormalities during followup visit. Some patients experience rapid increase in fluid volume, hemodynamic abnormalities, or aspiration difficulties during pericardial aspiration, thoracic surgery is needful when blood pressure continues to decline. Antibiotic therapy is required in some patients who develop chronic pericarditis after removal of the fluid. ${ }^{[9]}$

The occurrence of heart perforation is potentially life threatening and more difficult to handle. Therefore, it is especially important to prevent pacemaker related heart perforation in advance. Whether temporary pacing catheter was used before or during operation was one of the strongest risk factors, ${ }^{[10]}$ followed by the application of active fixation electrode wire, hormone, age, exposure time and body mass index $(<20)$. The reason that the use of temporary pacing catheters increased risk of perforation was associated with increased numbers of right ventricular leads, excellent catheters, or frequent emergency use. In conclusion, it is rare, but not uncommon and potentially dangerous thing that cardiac pacing leads to perforation of the heart, which should be taken seriously by clinicians. This disease is suspected when the pacing threshold increases, poor perception or cardiac $X$-ray imaging shows pulse electrode wire piercing epicardial margin of $3 \mathrm{~mm}$ above. Diagnosis can be based on ECG, intracavitary electrocardiogram, chest radiograph, echocardiography and chest CT and other tests. For early cardiac perforation, the pacing electrode leads can be removed by intravenous route with the support of ultrasound and cardiac surgery. For advanced electrode wire perforation, surgical thoracotomy may be required and care should 
be taken. The patients require more attention in the following cases: the use of temporary pacing electrodes or atrial active electrode electrodes, preoperative and postoperative use of hormones, older, too long exposure time, and body mass index $<20 .^{[11]}$ Only by paying great attention and fully realizing the risk and possibility of heart perforation can it be helpful for prevention, early diagnosis and effective treatment.

The patient had no apparent signs of electrode lead perforation during surgery, as the $X$-ray image was normal as well as pacemaker perception and pacing function. The causes of myocardial perforation third days after operation were analyzed: comparing the images left at the time of operation and the third day imaging, too long electrode wires were reserved and the local tension was too large. The ventricular pacing function of our case was lost but the sensory function was normal after electrode perforation. The pacemaker electrode of this patient was changed to bipolar programmed, and the pacemaker function returned to normal, indicating that circumpolar pacing was caused by electrode perforation. In order to avoid eventual perforation, pacing electrodes should be replaced in time though it turns out to be circumpolar pacing.

\section{Conflicts of Interest Disclosure}

The authors have no conflict of interest related to this article.

\section{References}

[1] Polin GM, Zado E, Nayak H, et al. Proper management of pericardial tamponade as a late complication of implantable cardiac device placement. American Journal of Cardiology. 2006; 98(2): 223-225. PMid: 16828597. https://doi.org/10.1016/ j.amjcard.2006.01.078

[2] Dillingboer D, Ector H, Willems R, et al. Pericardial effusion and right-sided pneumothorax resulting from an atrial active-fixation lead. Europace. 2003; 5(4): 419. https://doi.org/10.1016/ S1099-5129(03) 00079-5

[3] Danik SB, Mansour M, Singh J, et al. Increased incidence of subacute lead perforation noted with one implantable cardioverterdefibrillator. Heart Rhythm. 2007; 4(4): 439-442. PMid: 17399630. https://doi.org/10.1016/j.hrthm.2006.12.044

[4] Mortensen K, Aydin MA, Goldmann B, et al. Fluoroscopy to assess late heart and lung perforation by a permanent ventricular pacemaker lead. A case complicated by isolated hemothorax. International Journal of Cardiology. 2008; 128(1): 104106. PMid: 17706810. https://doi.org/10.1016/j.ijcard. 2007.04 .189

[5] Amara W, Cymbalista M, Sergent J. Delayed right ventricular perforation with a pacemaker lead into subcutaneous tissues. Archives of Cardiovascular Diseases. 2010; 113(1): 53-54. PMid: 20142121.

[6] Sakai Y, Sato Y, Matsuo S, et al. Perforation of the right ventricular free wall by an ICD lead in a patient with isolated noncompaction of the ventricular myocardium. International Journal of Cardilogy. 2007; 107(3): e104-e106.

[7] Crusio RH, Greenberg YJ. An unusual presentation of implantable cardioverter-defibrillator lead perforation. Journal of Electrocardiology. 2009; 42(3): 265-266. PMid: 18976778. https://doi.org/ 10.1016/j.jelectrocard.2008.09.005

[8] Ramirez MF, Ching CK, Ho KL, et al. "The attack of the $52 \mathrm{~cm}$ lead": an unusual case of late cardiac perforation by a passivefixation permanent pacemaker lead. International Journal of Cardiology. 2007; 115(1): 5-7. PMid:16901560. https://doi.org/10. 1016/j.ijcard.2006.05.064

[9] Geng RY, Zhu ZL, Hua W. Practical cardiac pacing technology. Beijing: People's Military Medical Publishing House; 2004. 267-270 p.

[10] Zou T, Wang ZL, Tong JB. Preliminary study of the cause of electrode dislocation in cardiac pacemaker. Chinese Journal of Cardiovascular Review. 2007; 5(6): 411-413.

[11] Guo JH, Xu Y, Li B, et al. Clinical study of lead dislocation of pacing electrode. Chinese Journal of Cardiac Pacing and Electrophysiology. 2002; 16(1): 8-10. 